\title{
Uncovering the inferior fronto-occipital fascicle and its topological organization in non- human primates: the missing connection for language evolution
}

Sarubbo, Silvio; Petit, Laurent; De Benedictis, Alessandro; Chioffi, Franco; Ptito, Maurice; Dyrby, Tim B.

Published in:

Brain Structure and Function

Link to article, DOI:

$10.1007 / \mathrm{s} 00429-019-01856-2$

Publication date:

2019

Document Version

Peer reviewed version

Link back to DTU Orbit

Citation (APA):

Sarubbo, S., Petit, L., De Benedictis, A., Chioffi, F., Ptito, M., \& Dyrby, T. B. (2019). Uncovering the inferior fronto-occipital fascicle and its topological organization in non-human primates: the missing connection for language evolution. Brain Structure and Function, 224(4), 1553-1567. https://doi.org/10.1007/s00429-01901856-2

\section{General rights}

Copyright and moral rights for the publications made accessible in the public portal are retained by the authors and/or other copyright owners and it is a condition of accessing publications that users recognise and abide by the legal requirements associated with these rights.

- Users may download and print one copy of any publication from the public portal for the purpose of private study or research.

- You may not further distribute the material or use it for any profit-making activity or commercial gain

- You may freely distribute the URL identifying the publication in the public portal 
archives-ouvertes

\section{Uncovering the inferior fronto-occipital fascicle and its topological organization in non-human primates: the missing connection for language evolution}

Silvio Sarubbo, Laurent Petit, Alessandro de Benedictis, Franco Chioffi, Maurice Ptito, Tim Dyrby

\section{To cite this version:}

Silvio Sarubbo, Laurent Petit, Alessandro de Benedictis, Franco Chioffi, Maurice Ptito, et al.. Uncovering the inferior fronto-occipital fascicle and its topological organization in non-human primates: the missing connection for language evolution. Brain Structure and Function, Springer Verlag, 2019, 224 (4), pp.1553-1567. 10.1007/s00429-019-01856-2 . hal-02063658

\section{HAL Id: hal-02063658 \\ https: / hal.archives-ouvertes.fr/hal-02063658}

Submitted on 13 Nov 2020

HAL is a multi-disciplinary open access archive for the deposit and dissemination of scientific research documents, whether they are published or not. The documents may come from teaching and research institutions in France or abroad, or from public or private research centers.
L'archive ouverte pluridisciplinaire HAL, est destinée au dépôt et à la diffusion de documents scientifiques de niveau recherche, publiés ou non, émanant des établissements d'enseignement et de recherche français ou étrangers, des laboratoires publics ou privés. 
Uncovering the inferior fronto-occipital fascicle and its topological organization in nonhuman primates: The missing connection for language evolution

\author{
Silvio Sarubbo ${ }^{{ }^{* \wedge}}$, Laurent Petit ${ }^{2 \wedge}$, Alessandro De Benedictis $^{3}$, \\ Franco Chioffi ${ }^{1}$, Maurice Ptito ${ }^{4}$, Tim B. Dyrby ${ }^{5,6}$.
}

${ }^{1}$ Division of Neurosurgery, Department of Emergency Medicine, Azienda Provinciale per i Servizi Sanitari (APSS) - 38122 Trento, Italy

${ }^{2}$ Groupe d'Imagerie Neurofonctionnelle, Institut des Maladies Neurodégénératives - UMR 5293, CNRS, CEA University of Bordeaux, Bordeaux, France

${ }^{3}$ Neurosurgery Unit, Department of Neuroscience and Neurorehabilitation, Bambino Gesù Children`s Hospital, IRCCS - 00165 Rome, Italy

${ }^{4}$ École d'optométrie, Université de Montréal, Montreal, QC, Canada

${ }^{5}$ Danish Research Centre for Magnetic Resonance, Center for Functional and Diagnostic Imaging and Research, Copenhagen University Hospital Hvidovre, Hvidovre, Denmark;

${ }^{6}$ Department of Applied Mathematics and Computer Science, Technical University of Denmark, Kongens Lyngby, Denmark

\title{
* Corresponding Author
}

Silvio Sarubbo, MD PhD

Division of Neurosurgery - Department of Emergency

Structural and Functional Connectivity Lab Project

"S. Chiara" Hospital, APSS Trento

9, Largo Medaglie d'Oro

38122 Trento (Italy)

e-mail: silviosarubbo@gmail.com

\section{${ }^{\wedge}$ These Authors contributed equally}




\begin{abstract}
Whether brain networks underlying the multimodal processing of language in humans are present in non-human primates is an unresolved question in primate evolution. Conceptual awareness in humans, which is the backbone of verbal and non-verbal semantic elaboration, involves intracerebral connectivity via the inferior fronto-occipital fascicle (IFOF). While nonhuman primates can communicate through visual information channels, there has been no formal demonstration that they possess a functional homologue of the human IFOF. Therefore, we undertook a post-mortem diffusion MRI tractography study in conjunction with Klingler micro-dissection to search for IFOF fiber tracts in brain of Old-World (vervet) monkeys. We found clear and concordant evidence from both techniques for the existence of bilateral fiber tracts connecting the frontal and occipital lobes. These tracts closely resembled the human IFOF with respect to trajectory, topological organization, and cortical terminal fields. Moreover, these fibers are clearly distinct from other bundles previously described in this region of monkey brain, i.e. the inferior longitudinal and uncinate fascicles, and the external and extreme capsules. This demonstration of an IFOF in brain of a species that diverged from the human lineage some 22 millions years ago enhances our comprehension about the evolution of language and social behavior.
\end{abstract}

Keywords: white matter, Klingler dissection, inferior fronto-occipital fascicle, brain connectivity, tractography, monkey anatomy 


\section{ETHICS STATEMENTS}

Authors have no conflict of interests and did not receive funding for this work.

The brain specimens were collected from animals enrolled in a terminal project reviewed and approved by the Institutional Review Board of the Behavioural Science Foundation (BSF; StKitts, West Indies) and were donated by Prof. Roberta Palmour of McGill University, in a collaboration with the BSF.

The human brain dissection presented in the paper was performed in the context of the Structural and Functional Connectivity Lab Project, approved by the Research Ethics Committee of the Azienda Provinciale dei Servizi Sanitari (APSS) of Trento (Italy).

\section{ACKNOWLEDGMENTS}

We are very grateful to the valuable work of proofreading of the present manuscript provided by Inglewood Biomedical Editing (www.inglewoodbiomedediting.com). 


\section{INTRODUCTION}

Conceptual awareness, or the sense of self is the foundation of semantics, language, and social behavior (Hurford 2003). This multi-level functional development is subserved in human brain by the so-called ventral (or "what") pathway, which encompasses several long white matter (WM) fasciculi: the uncinate (UF), inferior longitudinal (ILF), middle longitudinal (MdLF), and inferior fronto-occipital fascicles (IFOF) (Saur et al. 2008; Duffau 2015). Recent research has shown the IFOF to be the main structural pathway for verbal semantic elaboration in the language dominant hemisphere of human brain (Sarubbo et al. 2015a; Duffau 2015; Sarubbo et al. 2013). Deactivation of the IFOF in the non-dominant language hemisphere through direct electrical stimulation (DES) does not block speech, but perturbs non-verbal semantic cognition. This dissociation highlights the bilateral multimodal organization of the semantic network, which these long-range association fibers subserve (Herbet et al. 2017). Trolard and Curran first described the IFOF in human brain in the early $20^{\text {th }}$ century (Trolard 1906; Curran 1909), following upon an earlier description by Burdach (Burdach 1822). Micro-dissection and diffusion MRI tractography studies have recently confirmed the anatomy of this pathway (Hau et al. 2016; Sarubbo et al. 2013; Martino et al. 2010; Caverzasi et al. 2014; Wu et al. 2016; Panesar et al. 2017). Its entire course through the ventral part of the external capsule (EC) connects the occipital cortex and (to a lesser extent) the parietal and temporal cortices to the frontal cortex.

Like humans, non-human primates can communicate with conspecifics in the special sense of transforming visual information into vocalizations that may include semantic content. In fact, ethological studies established decades ago that Old-World monkeys, such as vervets, give alarm calls in response to viewing predators, produce distinct vocal warnings to different predators, and generally categorize other species by visual identification (Price et al. 2015; Seyfarth et al. 1980). To accomplish this, Old-World monkeys must engage a ventral WM 
pathway analogous to the human IFOF. Certainly, the processing of visual information in the primate ventral pathway must play a crucial role in semantic communication, especially so in Old-World monkeys, given the relatively large territory of their primary visual cortex compared to that of other primary senses (Schoenemann 2012). Moreover, neuronal tracttracing studies show that Old-World monkeys possess a ventrolateral frontal cortical area, which is architectonically and physiologically comparable to Broca's area, which subserves human speech production (Duffau et al. 2003; Petrides et al. 2005).

Previous authors proposed a structural substrate for the "what" pathway in non-human primates, distinct from the human IFOF in connecting only the temporal cortex to the inferior frontal cortex through the UF and the extreme capsule (EmC) (Petkov and Wilson 2012). There has hitherto been no documentation in monkey neuroanatomic studies of a pathway corresponding to the ventral part of the IFOF (Petkov and Wilson 2012; Schmahmann and Pandya 2006; Schmahmann et al. 2007). Although invasive tract-tracing studies are generally impossible in in vivo human brain, investigations of the IFOF have greatly benefited from the combination of ex-vivo Klingler micro-dissection and in-vivo tractography (Hau et al. 2016; Sarubbo et al. 2013; Martino et al. 2010). For the first time, we have applied this dual tract tracing method in a systematic search for the presence of IFOF in Old-World monkeys, aiming to establish the course and the cortical projections through ex-vivo diffusion MRI tractography combined with and Klingler micro-dissection of the same vervet monkey brains.

\section{METHODS}

\section{Subjects}

Two male/female vervet monkeys (Chlorocebus aethiops sabeus) aged 3.5-4 years were included in the neuroanatomic study combining ex vivo diffusion MRI followed by Klingler micro-dissection. The animals had been raised in an enriched environment in the 
laboratories of the Behavioral Science Foundation (BSF; St-Kitts, West Indies), a facility that is recognized by the Canadian Council on Animal Care (CCAC). The brain specimens were collected from animals enrolled in a terminal project reviewed and approved by the local Institutional Review Board of the BSF, and were donated by Prof. Roberta Palmour from McGill University, in a collaboration with the BSF. The animals were deeply sedated with ketamine hydrochloride (10 mg/kg, i.m.) intramuscular and then killed with an overdose of sodium pentobarbital ( $25 \mathrm{mg} / \mathrm{kg}$, i.v.), according to procedures approved by the American Veterinary Medical Association. After death, they were transcardially perfused with phosphate buffered saline (PBS-pH 7.4), followed by 4\% paraformaldehyde (PFA) solution in PBS (1 L). Following fixation in situ, the brains were carefully removed from the cranium and prepared for MRI.

\section{Ex-vivo diffusion MRI}

Diffusion MRI was acquired from the two perfusion-fixed monkey brains (M1 and M2) on a Bruker BioSpec 7 T MR scanner with maximal gradient of $660 \mathrm{mT} / \mathrm{m}$ according to an $e x-$ vivo imaging pipeline described previously (Dyrby et al. 2011). In short, to restore the T2 signal prior to MRI scanning, excess PFA was removed by immersing the brains in a large volume of PBS for at least one week. We ensured minimal susceptibility artifacts in dMRI and continued tissue hydration during the prolonged scanning by sealing the brains in individual plastic wrappers containing a small volume of PBS. The brains were then placed within a $72 \mathrm{~mm}$ quadrature radio frequency $(\mathrm{RF})$ coil for acquiring a pulsed-gradient spin echo (PGSE) sequence with single-line readout. Short-term instabilities due to thermal equilibration and small motion effects due to physical handling of the tissue were reduced by acquiring diffusion MRI dummy scans for at least five hours. The dMRI acquisition included 130 whole-brain image volumes repeated with a number of excitations (NEX), i.e. five for M1 and nine for M2. 
Ten image volumes were acquired at $b=0 \mathrm{~s} / \mathrm{mm}^{2}\left(b_{0}\right)$ and 128 volumes acquired with evenly distributed non-collinear gradient directions at nominally $\mathrm{b}=7,700 \mathrm{~s} / \mathrm{mm}^{2}$ (diffusion encoding pulse duration $(\delta)=8 \mathrm{~ms}$; time of onset between diffusion encoding gradients $(\Delta)=17 \mathrm{~ms}$, and gradient strength $(\mathrm{G})=350 \mathrm{mT} / \mathrm{m})$. The scan parameters were as follows: TR $=3061 \mathrm{~ms}, \mathrm{TE}$ $=31.4 \mathrm{~ms}$, matrix $128 \times 256,85$ slices, FOV $=64 \times 128 \mathrm{~mm}^{2}$, voxel size $=0.5 \times 0.5 \times 0.5 \mathrm{~mm}$. Total scan time per NEX was 16 hours, thus giving grant totals of 80 hours for M1 and 144 hours for M2. We saw a minor spatial drift in the dMRI dataset, which was corrected using the FMRIB software library (FSL, (Smith et al. 2004)).

\section{Tractography}

We used the streamline tractography framework that reduces tractography biases in position, shape, size, and length of delineated tracts (Girard et al. 2014). Whole-brain fiber peaks maps were first calculated from spherical deconvolution and then used for tractography (Tournier et al. 2007; Descoteaux et al. 2009) (Supplementary Figure 1b). Deterministic streamline tractography with reduced tracking biases was performed using the Continuous Map Criterion (CMC) and the Particle Filtering Tractography (PFT) method developed by Girard et al (2014) and implemented in Dipy (Garyfallidis et al. 2014). The CMC strategy uses WM, GM, and CSF partial volume estimation to define the probability of stopping the tracking process, whereas the PFT ensures robust tracking through complex anatomical regions. The default tractography parameters were maximal curvature of $60^{\circ}$ and a step-size of $0.05 \mathrm{~mm}$. Streamline lengths exceeding $90 \mathrm{~mm}$ or less than $10 \mathrm{~mm}$, as well as looping streamlines $(>360$ degrees), were excluded. 
Seeding, inclusion and exclusion masks

Seeding, inclusion and exclusion masks were needed for generating the whole-brain tractograms and to delineate association fibers. These were based on a publicly available postmortem rhesus macaque brain atlas (CIVM) (Calabrese et al. 2015). Both vervets (Chlorocebus pygerythrus, used in current study) and macaques (Macaca mulatta, CIVM template) are Old-World monkeys. Upon adjusting for minor brain size differences, the two templates match closely in shape (Martin and Bowden 2000; Maldjian et al. 2014). We warped the CIVM into the native spaces of the M1 and M2 T2w images by using ANTS linear and non-linear registration (stnava.github.io/ANTs, (Avants et al. 2011)). The final masks for tractography included WM, deep GM, and CSF masks, a midsagittal plane, and masks defining the entire right and left hemispheres. Six cortical lobar masks (frontal, occipital, parietal, temporal, cingulum and insula) were created for each hemisphere by grouping the respective cortical CIVM ROIs, and we used to delineate association fibers (Figure Fig. 1e).

\section{Generation of tractograms}

Whole-brain tractograms were generated by seeding 100 times each voxel within the WM mask, which resulted in more than two million streamlines in each animal. Spurious streamlines were excluded (i.e. those terminating within the lateral ventricles) using a CSF exclusion mask (Supplementary Figure 2a-b). Exclusion of streamlines not representing the association tracts of each hemisphere was accomplished by the following exclusion criteria: streamlines passing through the midbrain, those terminating in deep GM structures (basal ganglia and thalamus), and commissural streamlines passing through the inter-hemispheric midline, consisting on the main of callosal and anterior commissural streamlines (Supplementary Figure 2c-e). Then, we split the remaining association streamlines into left and right hemispheric tractograms (Supplementary Figure 2f). 
Fully automated virtual dissection of the IFOF, ILF, UF and EmC

Association fibers were segmented in each hemisphere based on their trajectory and connections between any two of the six cortical lobes. This resulted in six intra- and 15 interlobar sub-tractograms, from which four inter-lobar association bundles were segmented, namely the IFOF between the frontal and occipital lobes, the ILF between the temporal and occipital lobes, and the UF and EmC between the frontal and temporal lobes. The three ventral bundles were extracted based on their classical definition (Schmahmann and Pandya 2006; Schmahmann et al. 2007).

IFOF segmentation. To construct the IFOF, the medial-most streamlines passing through the cingulum were removed from the sub-tractogram encompassing all the streamlines with one termination in the occipital cortex and the other in the frontal cortex. It is worth noting that this simple segmentation process yielding fronto-occipital streamlines resembling the well-known IFOF course in humans.

ILF segmentation. The ILF was constructed from the temporo-occipital sub-tractogram by extracting all streamlines with one termination in the occipital cortex and the other in the ROIs corresponding to the superior temporal gyrus along the superior temporal sulcus in the CIVM template (see Calabrese et al. (2015) for ROI labeling).

UF and EmC segmentations. Both UF and EmC were extracted by first considering the subtractogram encompassing all streamlines with one termination in the frontal lobe and the other in the temporal lobe. We then applied the temporal CIVM ROIs to extract the fronto-temporal 
streamlines terminating in the temporo-polar CIVM ROIs as UF streamlines and those ending in the superior temporal gyrus and along the superior temporal sulcus as EmC.

Finally, we applied to each bundle the hierarchical QuickBundles clustering algorithm (Garyfallidis et al. 2014) to prune away outlier streamlines, namely any streamlines with an aberrant anatomical course (Côté et al. 2015). We applied the same $\alpha$ cut-off for the left and right hemispheres for IFOF ( $\alpha=0.8$ for M1, 0.6 for M2), ILF ( $\alpha=0.7$ for M1 and M2), and UF and EmC (( $\alpha=0.8$ for M1 and M2). Figure 3B shows in detail the patterns of these different bundles for one monkey (M1), whereas the bundle volumes for each monkey are presented in Table 1. All tractograms are visualized with the MI-Brain visualization software (www.imeka.ca/mi-brain) (Supplementary Figure 1d).

\section{Klingler's micro-dissection}

We processed the two brains by the Klingler method, according to the protocol previously described by our group (Sarubbo et al. 2016; De Benedictis et al. 2016; Sarubbo et al. 2015b; De Benedictis et al. 2014). After removal of the arachnoid mater, brains were immersed in a $10 \%$ formalin solution for 40 days at room temperature and then frozen at -80 ${ }^{\circ} \mathrm{C}$ for 20 days. Upon defrosting, we separated the two brains into four hemispheres. The microdissection (at $4 \mathrm{x}$ binocular magnification) consisted of initial removal of the gray matter of the occipital, parietal, temporal and frontal sulci to reveal the U-fibers, with particular attention to those connecting the occipital and temporo-parietal layers. This procedure exposed the fibers of the ILF, running inferiorly and anteriorly from the dorsal and ventral occipital cortices and the inferior parietal lobule to the middle and anterior portions of the temporal lobe. We subsequently exposed a layer of horizontal fibers directed to the whole occipital lobe, and continued with removal of the gray matter of the insula, thus exposing the fibers of the external capsule, which arch between the frontal and temporal opercula. These cortices were partially 
cut to expose the ventral third of the claustrum and external capsule, whereupon the residual gray matter of the claustrum was removed. This exposed a thick stem of WM including fibers arching between the frontal and the temporal poles (i.e. UF fibers) and fibers with a posteroanterior and superior course, connecting the temporal lobe to the frontal cortices. Following separation of the UF fibers and the isolation of the stem of the supposed IFOF, we proceeded to make a precise layer-by-layer dissection of the residual fibers with a postero-anterior and superior course extending their terminations at both ends.

We demarcated the main contingents of fibers in the various dissected bundles using thin colored tags to differentiate and visually highlight their reciprocal anatomical relationships (Figures 1-2 and 5-6). All steps of dissection were captured with high-definition images, which were stored for off-line analysis of the course, terminations, and anatomical relationships of the dissected bundles, as previously reported. The first author (S.S.) had earlier a performed corresponding dissection of the human IFOF in the context of the Structural and Functional Connectivity Lab Project (approved by the Ethical Committee) of the Azienda Provinciale dei Servizi Sanitari (APSS) of Trento (Italy). We present the human case along with the nonhuman primate preparations for the sake of comparison (Figure 1).

\section{RESULTS}

Discovery of monkey IFOF resembling the human IFOF

The MR tractography and Klingler micro-dissections both revealed the presence in each hemisphere of the monkey brains fiber bundles connecting the ventral part of the frontal cortex with the occipital cortex, running a ventral postero-anterior course similar to that of the human IFOF (Figure 1-4). Figure 1A shows in detail the matching anatomical features to tractography and microdissection. The rectilinear and fascicular segment of the frontal part of the IFOF was present in all four monkey brain hemispheres (labels 1-2). We also observed an upward- 
twisting enlargement of the bundle from the posterior output of the external capsule (labels 34), which coalesced into a large horizontal bundle projecting to the posterior-most part of the occipital lobe (labels 4-5). Of note, the same general pattern is discernible to tractography in vivo (Figures 1, 3 and 4).

We saw remarkable concordance between results of the successive micro-dissection steps exposing the IFOF, VOF, ILF, and UF with MR-based tractography findings of the same brain hemisphere (Figure 2). The tractography data enable the three-dimension depiction of the IFOF to an extraordinary degree of precision. Converging from the ventral part of the frontal lobe to form a narrow stem extending along the external capsule, the monkey IFOF progressively widens in a thin convex bundle of WM fibers running posteriorly up to the dorsal and ventral cortices of the occipital lobe (Figure 3A).

\section{Topological organization of the monkey IFOF}

Tractography revealed a topological organization of the IFOF in all four hemispheres examined (Figure 4). Concurring with the dissection results, these long-range fronto-occipital fibers terminate their anterior course in the ventro-lateral frontal cortices, while their posterior termination involves most of the occipital convexity. On other words, when emanating from the ventral part of the frontal cortex, the general topology of the IFOF retains its trajectory toward the occipital lobe. Occipital and frontal ROIs of the CIVM template were used to identify the distribution of the IFOF termination (Figure 4 and Table 2). In the four hemispheres examined, the occipital IFOF terminated mainly in the primary (V1), and to a lesser extent in the secondary visual area (V2). Those tractography streamlines ending in V2 consistently ran dorsally to the V1 streamlines and terminated mainly in the medial part of V2 (Figure 4A). The IFOF terminations in the ventral part of the frontal lobe were mainly distributed between lateral (areas 47 and premotor, orbital (areas 11) and polar (area 10) frontal cortices, and to a lesser 
extent in lateral area 46 and orbital area 14 (Figure 4B and Table 2). Results of the Klingler's micro-dissection indirectly support the MR tractography findings by showing a well-ordered fasciculation along the entire bundle. Figures 5 and 6 show more specifically that the different bundles (IFOF, UF and EmC) passing through the narrow region of external capsule do not cross and form a layer-based organization similar to the one observed in tractography on Figure 3B.

Monkey IFOF and the other ventral association fibers.

Both tractography and Klingler micro-dissection showed that anterior IFOF fibers of monkey brain were distinct from the UF and $\mathrm{EmC}$, with U-shaped courses between the frontal and temporal cortices. IFOF fibers posterior to ILF had a more infero-anterior directed course, extending from the dorsal and lateral occipital cortices to the anterior and basal temporal lobe (Figure 3B).

In comparison with the UF, the IFOF showed a more posterior direction and a longer trajectory to the occipital cortex (Figures 2, 3B and 5). Thus, the IFOF had a more dorsal and posterior location relative to the UF stem. The curvature of the uncinate fibers and the inverted triangular pattern of the IFOF bundle posterior to the UF were similar in tractography and micro-dissection of the same specimen. IFOF fibers were also clearly distinct from the EmC, as detailed in Figure 6. As in the dissection as usually performed for human UF/IFOF demonstration, we first highlighted the fibers of the EC and EmC (Figures 6B and 6C), which arch between the frontal and temporal opercula. These resided in a more superficial layer (Figure 6C, purple tag) of monkey WM compared to the fibers of the UF (Figure 6C, red tag). The stem of the UF lies deeper (Figure 6D, red tag) but also more ventral with respect to the EC, and has a distinctly different course and orientation (Figure 6D, bundle colored in light green) compared to the EC fibers (Figure 6D, upper bundle colored in light blue). After removal 
of the more superficial fibers of UF stem, we highlighted other fibers characterized by a longitudinal (Figure 6E, red tag) and antero-posterior course (Figure 6F, bundle colored in light green), in a layer lying beneath the fibers of the $\mathrm{EC} / \mathrm{EmC}$ (Figure $6 \mathrm{~F}$, raised by green pin). These fibers (Figure 6G, red tags), having a course similar to the human IFOF, showed a different orientation (Figure $6 \mathrm{H}$, bundle in light green) than the deeper and typically arching fibers of the monkey UF (Figure $6 \mathrm{H}$, bundle in purple). Finally, we revealed the full course of these fibers extending all the way to anterior the frontal lobe (Figure 6I).

\section{DISCUSSION}

In this study we used an integrated approach of ex-vivo diffusion-weighted tractography and Klingler micro-dissection for the first such investigation in the same Old-World monkey brain. We thus demonstrated the existence, course, topological organization, and cortical terminations of IFOF fiber connecting the occipital cortex with ventral frontal cortical territories of the vervet brain.

\section{The existence of the IFOF in the Old-World monkey brain}

Despite an abundance of functional evidence supporting a central role of occipitofrontal circuitry in cognition and sensory integration, previous neuroanatomic studies of the pathway in non-human primates using histological tracing or tractography (Schmahmann and Pandya 2006; Schmahmann et al. 2007) failed to demonstrate a direct connection between occipital and frontal cortices. The missing IFOF was notable, considering that those same monkey studies did reveal the other well-known association bundles of the ventral stream (ILF, UF, and EmC), which run parallel or around the human IFOF. In fact, there was recently a direct comparison by tractography of macaque and human EmC projections (Mars et al. 2016). 
Interestingly, that study indicated a surprising anatomic similarity of the macaque and human EmC pathways, suggesting a common inferior fronto-occipital pathway. Moreover, post mortem dissection of rhesus monkey brains recently gave the first direct evidence for the existence of IFOF in non-human primates (Decramer et al. 2018). We shall require a larger sample size to detect possible hemispheric asymmetries of the IFOF, as addressed in the human brain (Panesar et al. 2017; Wu et al. 2016; Hau et al. 2016). Thanks to the present combination of tractography and microdissection in the same specimens, we are also able to distinguish clearly the courses of the monkey EmC and IFOF (Figures 3 and 6).

Putting aside matters of terminology for the 'extreme capsule fiber complex' (Mars et al. 2016) versus IFOF, previous and present results call into question why earlier tracer studies failed to detect this long-range fronto-occipital pathway, despite attesting to the precise origin and termination of single constituent axons (Schmahmann and Pandya 2006). Earlier studies may have missed the monkey IFOF simply because it had not been their focus. Alternately, the pathway may not have been conspicuous in earlier tract tracing studies revealing only monosynaptic connections. That scenario would imply the existence of an indirect pathway from occipital to lateral/temporal cortex and thence to frontal cortex (through the EmC), rather than a direct fronto-occipital pathway. However, recent tracer studies did reveal sparse direct monosynaptic connections from frontal to visual areas of monkey brain, a matter calling for further investigation (Gerbella et al. 2010; Markov et al. 2014). Unfortunately, neither diffusion imaging tractography nor dissection can resolve the number of synapses in the pathway, due to their millimeter scaled resolution. Although the Klingler micro-dissection is an established complementary technique to combine with tractography data, its relative lack of quantitative measurements presents a limitation, compared to tractography, which yields bundle volumes and terminal fields. Generally, the challenge for quantification of micro-dissection is how to generate a distortion-free 3D volume of the dissection for projection in a space comparable to 
that of MRI. FIBRASCAN is a recently presented method that enables 3D imaging of stepwise dissection of a tract using a laser image scanner, and provides quantification of Klingler micro-dissection in conjunction with MRI (Zemmoura et al. 2014). Post-mortem brain photogrammetry has also recently emerged as a tool for post-dissection acquisition of accurate metrics of WM anatomy (De Benedictis et al. 2018). Applying brain photogrammetry during the different steps of WM micro-dissection can be combined with equivalent tractography data projected together on the 3D-brain rendering of the specimen. Such an approach promises to provide both qualitative and quantitative cross-validation between the two techniques, while quantifying structural brain connectivity. However, only tract-tracing studies in monkeys will definitively evaluation the contribution of monosynaptic fibers to the monkey IFOF in the light of precise inter-species homologies. Thus, the historical definition of IFOF in dissected human brain (Curran 1909), reinforced by recent tractography and microdissection data (Wu et al. 2016; Hau et al. 2016; Caverzasi et al. 2014; Sarubbo et al. 2013; Martino et al. 2010; Panesar et al. 2017), together substantiate the existence of the monkey IFOF. To quote Curan`s original description of the IFOF (1909 “...subsequent investigation brought out its true nature, that of continuous fibers uninterrupted by nuclei at any place in its course."

Our description of the monkey IFOF topological organization is consistent with the IFOF subdivision recently described in humans (Panesar et al. 2017; Wu et al. 2016; Hau et al. 2016). We observed that the ventro-lateral (area 47 and proMotor) and ventro-medial (area 10 and 11) frontal origins of the IFOF streamlines were topologically conserved along the bundle, with the former projecting to the medial occipital cortex and the later preferentially to a more territory (Figure 4B). As suggested by Panesar et al. (2017), such subdivided IFOF arrangements may subserve a multifunctional role of this bundle, where one division may be engaged in vocalization in response to visual cues. 
In general, functional evolution has strong links with structural evolution. Increasing gyrification and maturation of short connections are the basic elements of the phylogenetic evolution from non-human primate to human brain (Hofman 2012). These structural processes together enhance the capacity for multimodal integration and plasticity, and enable greater efficiency for brains with larger associative areas (Hofman 2012; Harrison et al. 2002; Karbowski 2003). Consequently, from an evolutionary perspective, one might not have expected that a long association pathway such as the IFOF should arise in human brain without earlier precedence established in the primate lineage.

Our linking of post-mortem diffusion-based tractography and Klinger's microdissection in the same specimens enabled the first demonstration of this crucial ventral pathway in non-human primates. The combination of high-sensitivity provided by tractography and specificity from direct visualization and isolation of WM bundles with Klingler's dissection was critical for the reliability of present results. Our cross-modal results concurred with respect to the IFOF course and its cortical termination, while also revealing the surrounding tracts previously described in monkeys (ILF, UF, EmC) around the external capsule. The external capsule embodies a known bottleneck in the WM bundles, which poses many problems to tractography algorithms and often leads to construction of false-positive bundles (Maier-Hein et al. 2017). Thanks to our combined approach in the same specimens, we observed by tractography and by Klingler's dissection that these different bundles showed a layer-based organization in the bottleneck region of the external capsule. Such laminar anatomical knowledge may be integrated as anatomical priors in advanced tractography algorithms to control for false-positive and false-negative bundle reconstruction (Daducci et al. 2016; Rheault et al. 2019; Innocenti et al. 2019; Dyrby et al. 2018). 


\section{The missing connection for language evolution}

Early efforts to establish intracranial structures relevant to the evolution of human language focused on asymmetry of the planum temporale in non-human primates (Gannon et al. 1998). However, the ventral network mediating the integrating sensory inputs with executive functions is a key issue from the functional perspective. Indeed, the mid-ventrolateral frontal territories of the human brain interact functionally with posterior cortical association areas in subserving the active retrieval of information required for basic decision processes, especially if relevant stimuli are not stably or strongly represented in memory (Petrides 2002). The monkey IFOF termination fields were ventral to the ventral premotor area (F5), where auditory and visual mirror neurons were originally described (Kohler et al. 2002). This territory lies anterior to the monkey cortical region that is cytoarchitectonically and neurophysiologically comparable to the human BA 44 region (Petrides et al. 2005) crucial for language production (Duffau et al. 2003; Ojemann et al. 1989).

Our present findings seem crucial for a reliable elucidation of the anatomical background of the dissociated and parallel processing of visual information by non-human primates. Indeed, the ventral stream connecting the occipital and frontal lobes underlies visual recognition and discrimination, which is a prerequisite for communicating threats to conspecifics. Particularly, ventro-lateral frontal cortices, which have functional interconnections with visual stream areas, are involved in processing pattern, color, object, and face information (Wilson et al. 1993). Moreover, the activation of perceptual representations may deliver (top-down) feedback effect from frontal cortices to the posterior visual areas (Ungerleider et al. 1998). Finally, a ventral pathway for proto-syntactic learning in non-human primates has been hypothesized, although not yet defined structurally (Petkov et al. 2008). 


\section{Conclusion}

The present study demonstrates the existence in Old-World monkeys of a long-range WM ventral pathway resembling that in humans, which seems poised to mediate the processing and integration of visual information for conceptualization and basic communication acts. This is the first multimodal demonstration of the existence in monkey brain of an anatomic structure deemed essential for conceptualization, and potentially supporting a core ability for semantic elaboration, comprehension and syntax communication in monkeys. Our results shed new light on the anatomical evolution of the ventral pathway in the primate lineage, constituting a missing link for understanding the evolution of language and social behavior in a brain system established more than 20 million years ago (Glazko and Nei 2003). 


\section{REFERENCES}

Avants BB, Tustison NJ, Wu J, Cook PA, Gee JC (2011) An open source multivariate framework for n-tissue segmentation with evaluation on public data. Neuroinformatics 9 (4):381-400. doi:10.1007/s12021-011-9109-y

Burdach KF (1822) Vom Baue und Leben des Gehirns. Dyk, Leipzig

Calabrese E, Badea A, Coe CL, Lubach GR, Shi Y, Styner MA, Johnson GA (2015) A diffusion tensor MRI atlas of the postmortem rhesus macaque brain. NeuroImage 117:408-416. doi:http://dx.doi.org/10.1016/j.neuroimage.2015.05.072

Caverzasi E, Papinutto N, Amirbekian B, Berger MS, Henry RG (2014) Q-Ball of Inferior Fronto-Occipital Fasciculus and Beyond. PLoS ONE 9 (6):e100274. doi:10.1371/journal.pone.0100274

Côté M-A, Garyfallidis E, Larochelle H, Descoteaux M Cleaning up the mess: tractography outlier removal using hierarchical QuickBundles clustering. In: 23rd ISMRM Annual Meeting, Toronto, Canada, 2015. vol 2844.

Curran EJ (1909) A new association fiber tract in the cerebrum with remarks on the fiber tract dissection method of studying the brain. Journal of Comparative Neurology and Psychology 19 (6):645-656. doi:10.1002/cne.920190603

Daducci A, Dal Palu A, Descoteaux M, Thiran JP (2016) Microstructure Informed Tractography: Pitfalls and Open Challenges. Front Neurosci 10 (247):247. doi:10.3389/fnins.2016.00247

De Benedictis A, Duffau H, Paradiso B, Grandi E, Balbi S, Granieri E, Colarusso E, Chioffi F, Marras CE, Sarubbo S (2014) Anatomo-functional study of the temporo-parietooccipital region: dissection, tractographic and brain mapping evidence from a neurosurgical perspective. J Anat 225 (2):132-151. doi:10.1111/joa.12204

De Benedictis A, Nocerino E, Menna F, Remondino F, Barbareschi M, Rozzanigo U, Corsini F, Olivetti E, Marras CE, Chioffi F, Avesani P, Sarubbo S (2018) Photogrammetry of the Human Brain: A Novel Method for Three-Dimensional Quantitative Exploration of the Structural Connectivity in Neurosurgery and Neurosciences. World Neurosurg 115:e279-e291. doi:10.1016/j.wneu.2018.04.036

De Benedictis A, Petit L, Descoteaux M, Marras CE, Barbareschi M, Corsini F, Dallabona M, Chioffi F, Sarubbo S (2016) New insights in the homotopic and heterotopic connectivity of the frontal part of the human corpus callosum revealed by microdissection and diffusion tractography. Hum Brain Mapp 37 (12):4718-4735

Decramer T, Swinnen S, van Loon J, Janssen P, Theys T (2018) White matter tract anatomy in the rhesus monkey: a fiber dissection study. Brain Struct Funct. doi:10.1007/s00429018-1718-x

Descoteaux M, Deriche R, Knosche TR, Anwander A (2009) Deterministic and probabilistic tractography based on complex fibre orientation distributions. IEEE transactions on medical imaging 28 (2):269-286. doi:10.1109/TMI.2008.2004424

Duffau H (2015) Stimulation mapping of white matter tracts to study brain functional connectivity. Nat Rev Neurol 11 (5):255-265. doi:10.1038/nrneurol.2015.51

Duffau H, Capelle L, Denvil D, Gatignol P, Sichez N, Lopes M, Sichez J-P, Van Effenterre R (2003) The role of dominant premotor cortex in language: a study using intraoperative functional mapping in awake patients. NeuroImage 20 (4):1903-1914. doi:https://doi.org/10.1016/S1053-8119(03)00203-9

Dyrby TB, Baaré WFC, Alexander DC, Jelsing J, Garde E, Søgaard LV (2011) An ex vivo imaging pipeline for producing high-quality and high-resolution diffusion-weighted imaging datasets. Human Brain Mapping 32 (4):544-563. doi:10.1002/hbm.21043 
Dyrby TB, Innocenti GM, Bech M, Lundell H (2018) Validation strategies for the interpretation of microstructure imaging using diffusion MRI. NeuroImage 182:62-79. doi:https://doi.org/10.1016/j.neuroimage.2018.06.049

Gannon PJ, Holloway RL, Broadfield DC, Braun AR (1998) Asymmetry of chimpanzee planum temporale: Humanlike pattern of Wernicke's brain language area homolog. Science 279:220-222

Garyfallidis E, Brett M, Amirbekian B, Rokem A, van der Walt S, Descoteaux M, NimmoSmith I, Dipy C (2014) Dipy, a library for the analysis of diffusion MRI data. Front Neuroinform 8:8. doi:10.3389/fninf.2014.00008

Gerbella M, Belmalih A, Borra E, Rozzi S, Luppino G (2010) Cortical connections of the macaque caudal ventrolateral prefrontal areas 45A and 45B. Cereb Cortex 20 (1):141168. doi:10.1093/cercor/bhp087

Girard G, Whittingstall K, Deriche R, Descoteaux M (2014) Towars quantitative connectivity analysis: Reducing tractography biaises. NeuroImage 98:266-278

Glazko GV, Nei M (2003) Estimation of divergence times for major lineages of primate species. Mol Biol Evol 20 (3):424-434. doi:10.1093/molbev/msg050

Harrison KH, Hof PR, Wang SS-H (2002) Scaling laws in the mammalian neocortex: Does form provide clues to function? Journal of Neurocytology 31 (3):289-298. doi:10.1023/a:1024178127195

Hau J, Sarubbo S, Perchey G, Crivello F, Zago L, Mellet E, Jobard G, Joliot M, Mazoyer B, Tzourio-Mazoyer N, Petit L (2016) Cortical terminations of the inferior fronto-occipital and uncinate fasciculi: Stem-based anatomical virtual dissection Front Neuroanat 10:58

Herbet G, Moritz-Gasser S, Duffau H (2017) Direct evidence for the contributive role of the right inferior fronto-occipital fasciculus in non-verbal semantic cognition. Brain Structure and Function 222 (4):1597-1610. doi:10.1007/s00429-016-1294-x

Hofman MA (2012) Chapter 18 - Design principles of the human brain: An evolutionary perspective. In: Michel AH, Dean F (eds) Progress in Brain Research, vol Volume 195. Elsevier, pp 373-390. doi:https://doi.org/10.1016/B978-0-444-53860-4.00018-0

Hurford JR (2003) The language mosaic and its evolution. In: Christiaens M, Kirby S (eds) Language Evolution. Oxford University Press, pp 38-57

Innocenti GM, Dyrby TB, Girard G, St-Onge E, Thiran JP, Daducci A, Descoteaux M (2019) Topological principles and developmental algorithms might refine diffusion tractography. Brain Struct Funct 224 (1):1-8. doi:10.1007/s00429-018-1759-1

Karbowski J (2003) How Does Connectivity Between Cortical Areas Depend on Brain Size? Implications for Efficient Computation. Journal of Computational Neuroscience 15 (3):347-356. doi:10.1023/a:1027467911225

Kohler E, Keysers C, Umilta MA, Fogassi L, Gallese V, Rizzolatti G (2002) Hearing sounds, understanding actions: action representation in mirror neurons. Science 297 (5582):846-848

Maier-Hein KH, Neher PF, Houde JC, Cote MA, Garyfallidis E, Zhong J, Chamberland M, Yeh FC, Lin YC, Ji Q, Reddick WE, Glass JO, Chen DQ, Feng Y, Gao C, Wu Y, Ma J, Renjie H, Li Q, Westin CF, Deslauriers-Gauthier S, Gonzalez JOO, Paquette M, StJean S, Girard G, Rheault F, Sidhu J, Tax CMW, Guo F, Mesri HY, David S, Froeling M, Heemskerk AM, Leemans A, Bore A, Pinsard B, Bedetti C, Desrosiers M, Brambati S, Doyon J, Sarica A, Vasta R, Cerasa A, Quattrone A, Yeatman J, Khan AR, Hodges W, Alexander S, Romascano D, Barakovic M, Auria A, Esteban O, Lemkaddem A, Thiran JP, Cetingul HE, Odry BL, Mailhe B, Nadar MS, Pizzagalli F, Prasad G, Villalon-Reina JE, Galvis J, Thompson PM, Requejo FS, Laguna PL, Lacerda LM, Barrett R, Dell'Acqua F, Catani M, Petit L, Caruyer E, Daducci A, Dyrby TB, HollandLetz T, Hilgetag CC, Stieltjes B, Descoteaux M (2017) The challenge of mapping the 
human connectome based on diffusion tractography. Nat Commun 8 (1):1349. doi:10.1038/s41467-017-01285-x

Maldjian JA, Daunais JB, Friedman DP, Whitlow CT (2014) Vervet MRI Atlas and Label Map for Fully Automated Morphometric Analyses. Neuroinformatics 12 (4):543-550. doi:10.1007/s12021-014-9231-8

Markov NT, Ercsey-Ravasz MM, Ribeiro Gomes AR, Lamy C, Magrou L, Vezoli J, Misery P, Falchier A, Quilodran R, Gariel MA, Sallet J, Gamanut R, Huissoud C, Clavagnier S, Giroud P, Sappey-Marinier D, Barone P, Dehay C, Toroczkai Z, Knoblauch K, Van Essen DC, Kennedy H (2014) A Weighted and Directed Interareal Connectivity Matrix for Macaque Cerebral Cortex. Cerebral Cortex 24 (1):17-36. doi:10.1093/cercor/bhs270

Mars RB, Foxley S, Verhagen L, Jbabdi S, Sallet J, Noonan MP, Neubert FX, Andersson JL, Croxson PL, Dunbar RI, Khrapitchev AA, Sibson NR, Miller KL, Rushworth MF (2016) The extreme capsule fiber complex in humans and macaque monkeys: a comparative diffusion MRI tractography study. Brain Struct Funct 221 (8):4059-4071. doi:10.1007/s00429-015-1146-0

Martin RF, Bowden DM (2000) Primate brain maps: Structure of the macaque brain. Elsevier Science, University of Washington

Martino J, Brogna C, Robles SG, Vergani F, Duffau H (2010) Anatomic dissection of the inferior fronto-occipital fasciculus revisited in the lights of brain stimulation data. Cortex 46 (5):691-699. doi:10.1016/j.cortex.2009.07.015

Ojemann G, Ojemann J, Lettich E, Berger M (1989) Cortical language localization in left, dominant hemisphere. An electrical stimulation mapping investigation in 117 patients. J Neurosurg 71 (3):316-326. doi:10.3171/jns.1989.71.3.0316

Panesar SS, Yeh F-C, Deibert CP, Fernandes-Cabral D, Rowthu V, Celtikci P, Celtikci E, Hula WD, Pathak S, Fernández-Miranda JC (2017) A diffusion spectrum imaging-based tractographic study into the anatomical subdivision and cortical connectivity of the ventral external capsule: uncinate and inferior fronto-occipital fascicles. Neuroradiology 59 (10):971-987. doi:10.1007/s00234-017-1874-3

Petkov CI, Kayser C, Steudel T, Whittingstall K, Augath M, Logothetis NK (2008) A voice region in the monkey brain. Nat Neurosci 11 (3):367-374

Petkov CI, Wilson B (2012) On the pursuit of the brain network for proto-syntactic learning in non-human primates: conceptual issues and neurobiological hypotheses. Philos Trans R Soc Lond B Biol Sci 367 (1598):2077-2088. doi:10.1098/rstb.2012.0073

Petrides M (2002) The Mid-ventrolateral Prefrontal Cortex and Active Mnemonic Retrieval. Neurobiology of Learning and Memory 78 (3):528-538. doi:https://doi.org/10.1006/nlme.2002.4107

Petrides M, Cadoret G, Mackey S (2005) Orofacial somatomotor responses in the macaque monkey homologue of Broca's area. Nature 435 (7046):1235-1238. doi:http://www.nature.com/nature/journal/v435/n7046/suppinfo/nature03628_S1.html

Price T, Wadewitz P, Cheney D, Seyfarth R, Hammerschmidt K, Fischer J (2015) Vervets revisited: A quantitative analysis of alarm call structure and context specificity. Scientific Reports 5:13220. doi:10.1038/srep13220

https://www.nature.com/articles/srep13220\#supplementary-information

Rheault F, St-Onge E, Tzourio-Mazoyer N, Sidhu J, Petit L, Descoteaux M (2019) BundleSpecific Tractography: Enhancing fiber tracking with additional anatomical and orientational priors. NeuroImage 186:382-398

Sarubbo S, De Benedictis A, Maldonado IL, Basso G, Duffau H (2013) Frontal terminations for the inferior fronto-occipital fascicle: anatomical dissection, DTI study and 
functional considerations on a multi-component bundle. Brain structure \& function 218:21-37. doi:10.1007/s00429-011-0372-3

Sarubbo S, De Benedictis A, Merler S, Mandonnet E, Balbi S, Granieri E, Duffau H (2015a) Towards a functional atlas of human white matter. Hum Brain Mapp 36 (8):3117-3136. doi: $10.1002 / \mathrm{hbm} .22832$

Sarubbo S, De Benedictis A, Merler S, Mandonnet E, Barbareschi M, Dallabona M, Chioffi F, Duffau H (2016) Structural and functional integration between dorsal and ventral language streams as revealed by blunt dissection and direct electrical stimulation. Hum Brain Mapp 37 (11):3858-3872. doi:10.1002/hbm.23281

Sarubbo S, De Benedictis A, Milani P, Paradiso B, Barbareschi M, Rozzanigo U, Colarusso E, Tugnoli V, Farneti M, Granieri E, Duffau H, Chioffi F (2015b) The course and the anatomo-functional relationships of the optic radiation: a combined study with 'post mortem' dissections and 'in vivo' direct electrical mapping. J Anat 226 (1):47-59. doi:10.1111/joa.12254

Saur D, Kreher BW, Schnell S, Kummerer D, Kellmeyer P, Vry MS, Umarova R, Musso M, Glauche V, Abel S, Huber W, Rijntjes M, Hennig J, Weiller C (2008) Ventral and dorsal pathways for language. Proc Natl Acad Sci U S A 105 (46):18035-18040. doi:10.1073/pnas.0805234105

Schmahmann JD, Pandya DN (2006) Fiber pathways of the brain. Oxford University Press, New York

Schmahmann JD, Pandya DN, Wang R, Dai G, D'Arceuil HE, de Crespigny AJ, Wedeen VJ (2007) Association fibre pathways of the brain: parallel observations from diffusion spectrum imaging and autoradiography. Brain 130 (3):630-653. doi:10.1093/brain/awl359

Schoenemann PT (2012) Chapter 22 - Evolution of brain and language. In: Michel AH, Dean F (eds) Progress in Brain Research, vol Volume 195. Elsevier, pp 443-459. doi:https://doi.org/10.1016/B978-0-444-53860-4.00022-2

Seyfarth R, Cheney D, Marler P (1980) Monkey responses to three different alarm calls: evidence of predator classification and semantic communication. Science 210 (4471):801-803. doi:10.1126/science.7433999

Smith SM, Jenkinson M, Woolrich MW, Beckmann CF, Behrens TEJ, Johansen-Berg H, Bannister PR, De Luca M, Drobnjak I, Flitney DE, Niazy RK, Saunders J, Vickers J, Zhang Y, De Stefano N, Brady JM, Matthews PM (2004) Advances in functional and structural MR image analysis and implementation as FSL. NeuroImage 23, Supplement 1 (0):S208-S219. doi:10.1016/j.neuroimage.2004.07.051

Tournier JD, Calamante F, Connelly A (2007) Robust determination of the fibre orientation distribution in diffusion MRI: Non-negativity constrained super-resolved spherical deconvolution. $\quad$ NeuroImage $\quad 35 \quad$ (4):1459-1472. doi:http://dx.doi.org/10.1016/j.neuroimage.2007.02.016

Trolard P (1906) Le faisceau longitudinal inférieur du cerveau. Revue Neurologique 14:440446

Ungerleider LG, Courtney SM, Haxby JV (1998) A neural system for human visual working memory. ProcNatlAcadSciUSA 95:883-890

Wilson FAW, Scalaidhe SPO, Goldman-Rakic PS (1993) Dissociation of object and spatial processing domains in primate prefrontal cortex. Science 260:1955-1958

Wu Y, Sun D, Wang Y, Wang Y (2016) Subcomponents and connectivity of the inferior frontooccipital fasciculus revealed by diffusion spectrum imaging fiber tracking. Frontiers in Neuroanatomy 10:88. doi:10.3389/fnana.2016.00088

Zemmoura I, Serres B, Andersson F, Barantin L, Tauber C, Filipiak I, Cottier JP, Venturini G, Destrieux C (2014) FIBRASCAN: a novel method for 3D white matter tract 
reconstruction in MR space from cadaveric dissection. Neuroimage 103:106-118. doi:10.1016/j.neuroimage.2014.09.016 


\section{FIGURE LEGENDS}

Figure 1. A. Results of microdissection and fiber tracking of the IFOF in two female vervet monkeys. B. Corresponding examples of Klingler's micro-dissection and tracking of IFOF fibers $^{12,22}$ in the left human hemisphere ex vivo and in vivo, respectively. The tractography reconstructions of the human IFOF were made using the method of Hau et al. (2016).

Figure 2. Upper: the layer-by-layer dissection (with Klingler's technique on the left and tractography on the right) of the lateral WM at the temporo-occipital region is shown, along with demarcation of vertical fibers of the VOF (red tags, blue fibers). Middle: a depiction of the crossing course between the vertical fibers of the ILF and the posterior portion of the IFOF. Bottom: an overview of the course and relationships between the IFOF (green), running within the ventral third of the external capsule, the parallel and more inferior course of the ventral portion of the ILF (red) and the arching fibers of the UF (yellow), running paired to the IFOF. IFOF: inferior fronto-occipital fascicle; ILF: inferior longitudinal fascicle; UF: uncinate fascicle; VOF: vertical occipital fascicle.

Figure 3. A. Different three-dimensional views of the left and right IFOF of a vervet monkey brain (M1) determined by tractography ex vivo. A: anterior view. B. tractography overview of the anatomical relationships between the inferior fronto-occipital fascicle (IFOF, green fibers) and the other ventral pathways in the same monkey brain (M1), including inferior longitudinal fascicles (ILF, red fibers), uncinate fascicle (UF, yellow fibers), and extreme capsule (EmC, blue fibers).

Figure 4. Three-dimensional views of the left and right IFOF of the two vervet monkeys (M1 and M2) showing the distributions of the occipital (A) and frontal (B) terminations. See Table 2 for the proportional volumes. A: anterior, L: left and R: right.

Figure 5. Micro-dissection and tractography details highlighting the main differences between the UF and IFOF stems in monkey brain. See text in Results for details.

Figure 6. Details of the microdissection highlighting the main differences between the fibers of UF/IFOF stem and the well-known fibers of the EmC, as emerged after removal of the fronto-temporal opercula and insular cortex (A) by the Klingler method. See text in Results for details.

A: anterior; EC: external capsule; EmC: extreme capsule; IFOF: inferior fronto-occipital fascicle; P: posterior; UF: uncinate fascicle. 


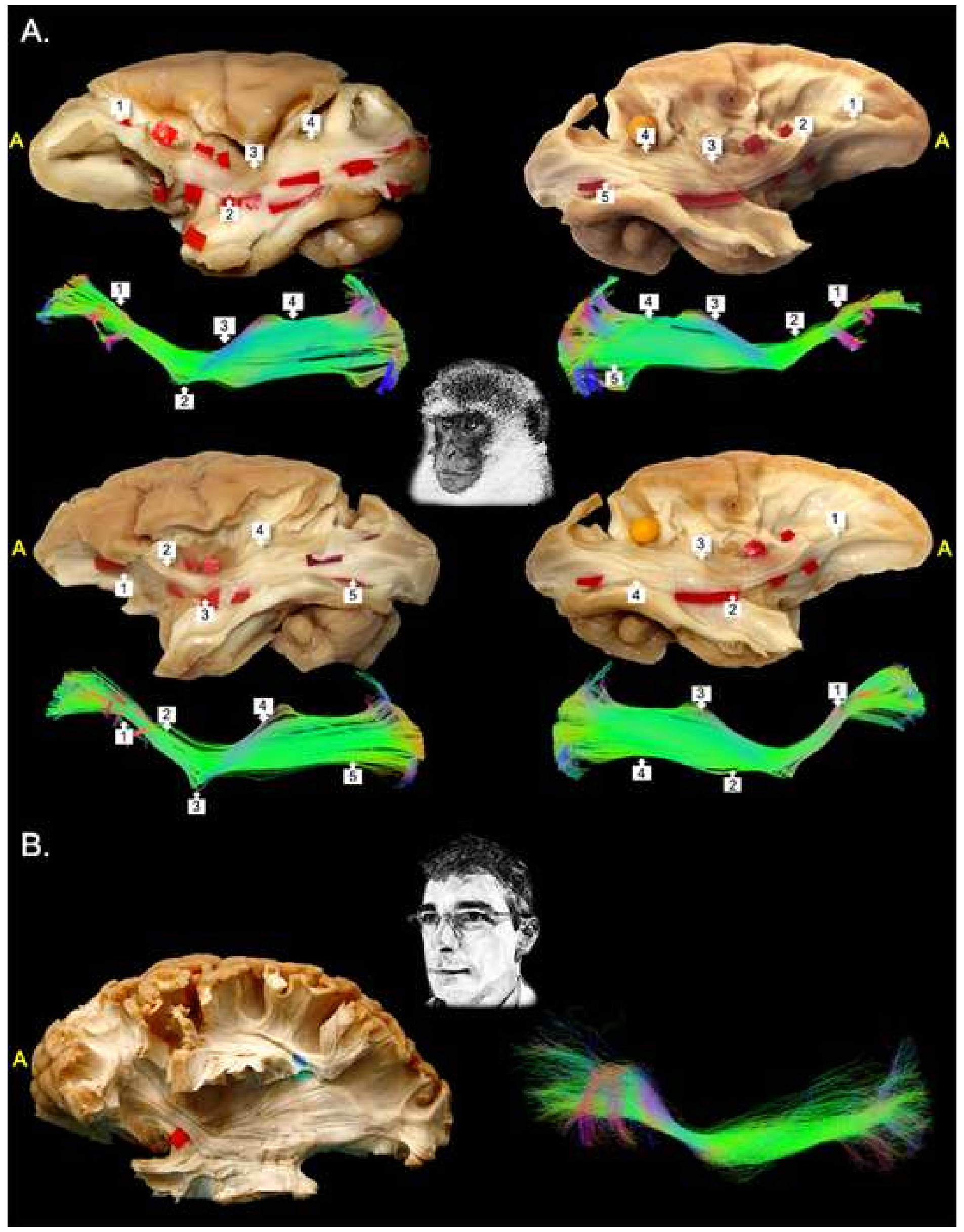



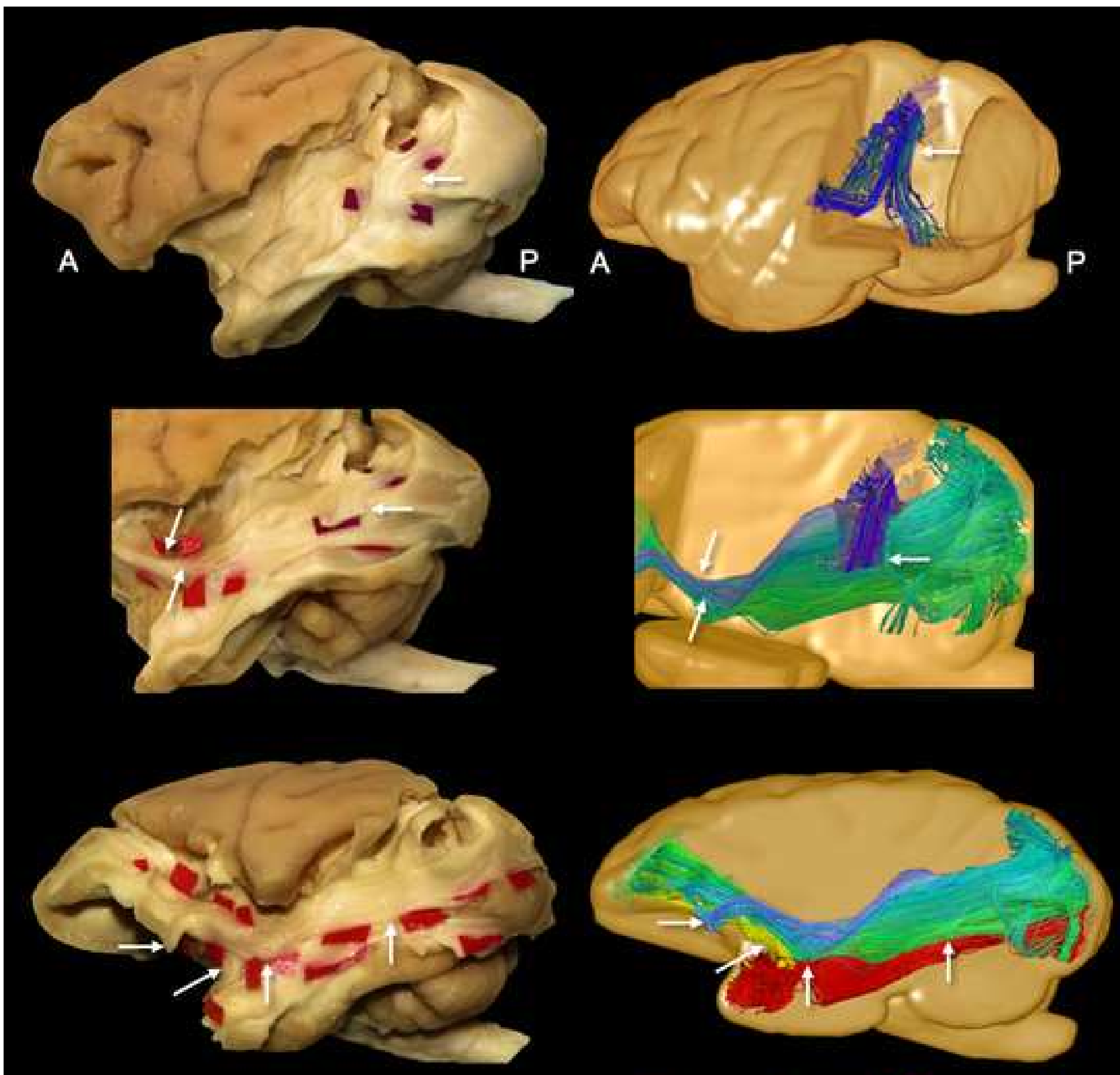

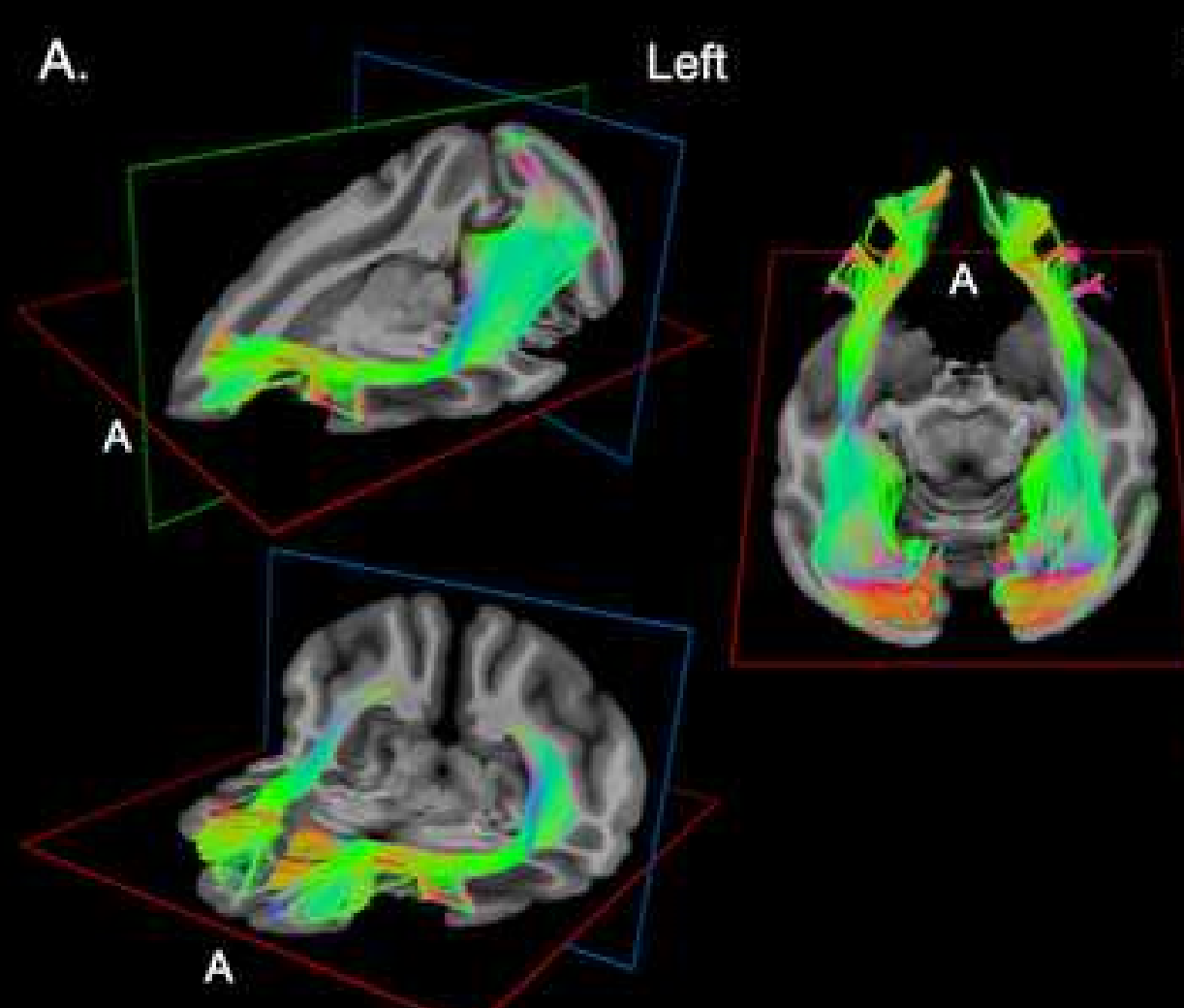

Right
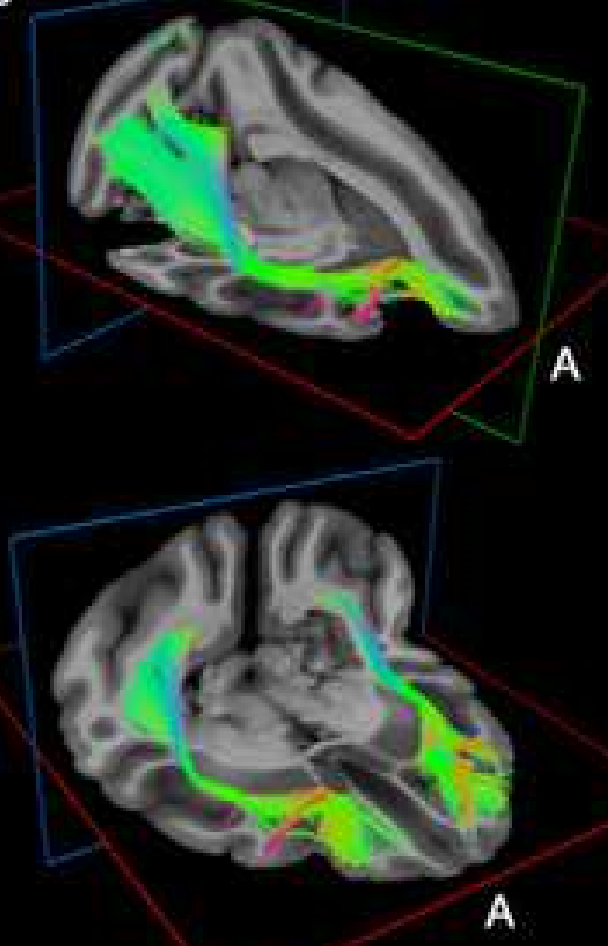

B.

Left
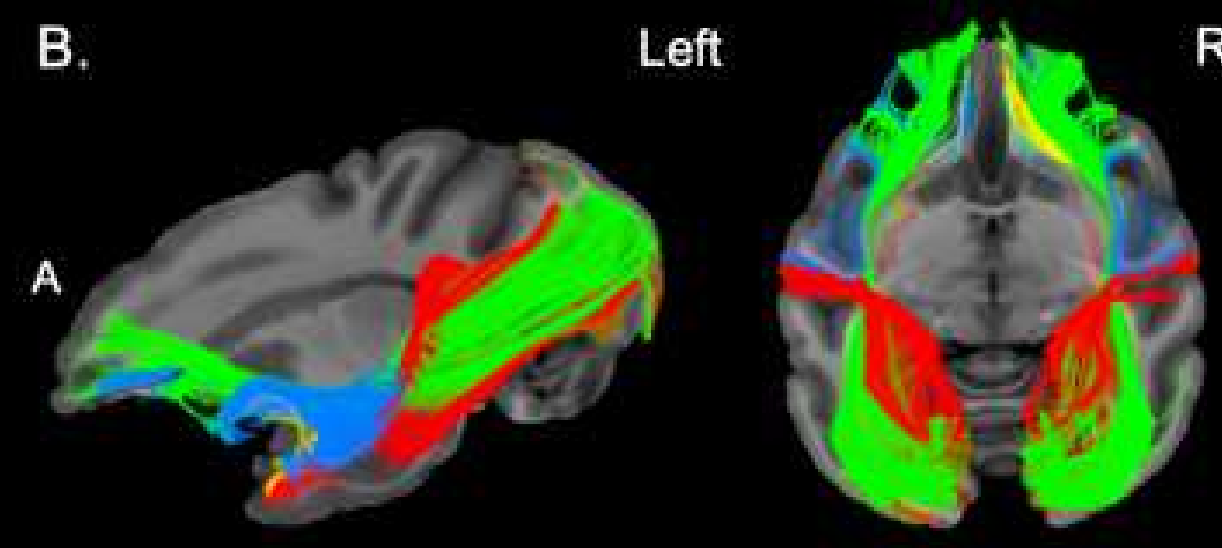

Right

IFOF

IILF $\square$ UF

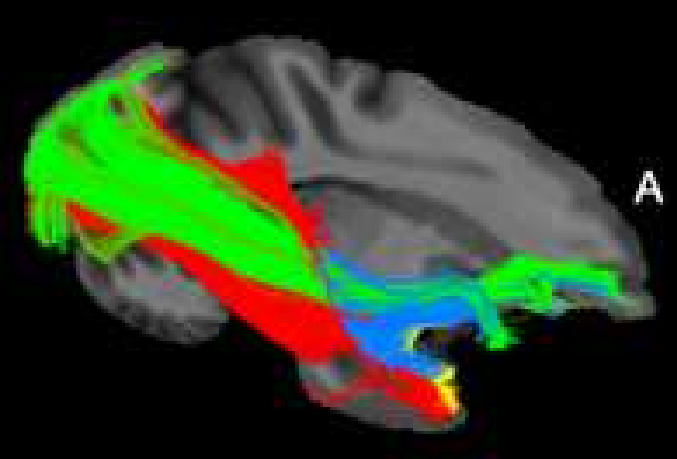

EmC 


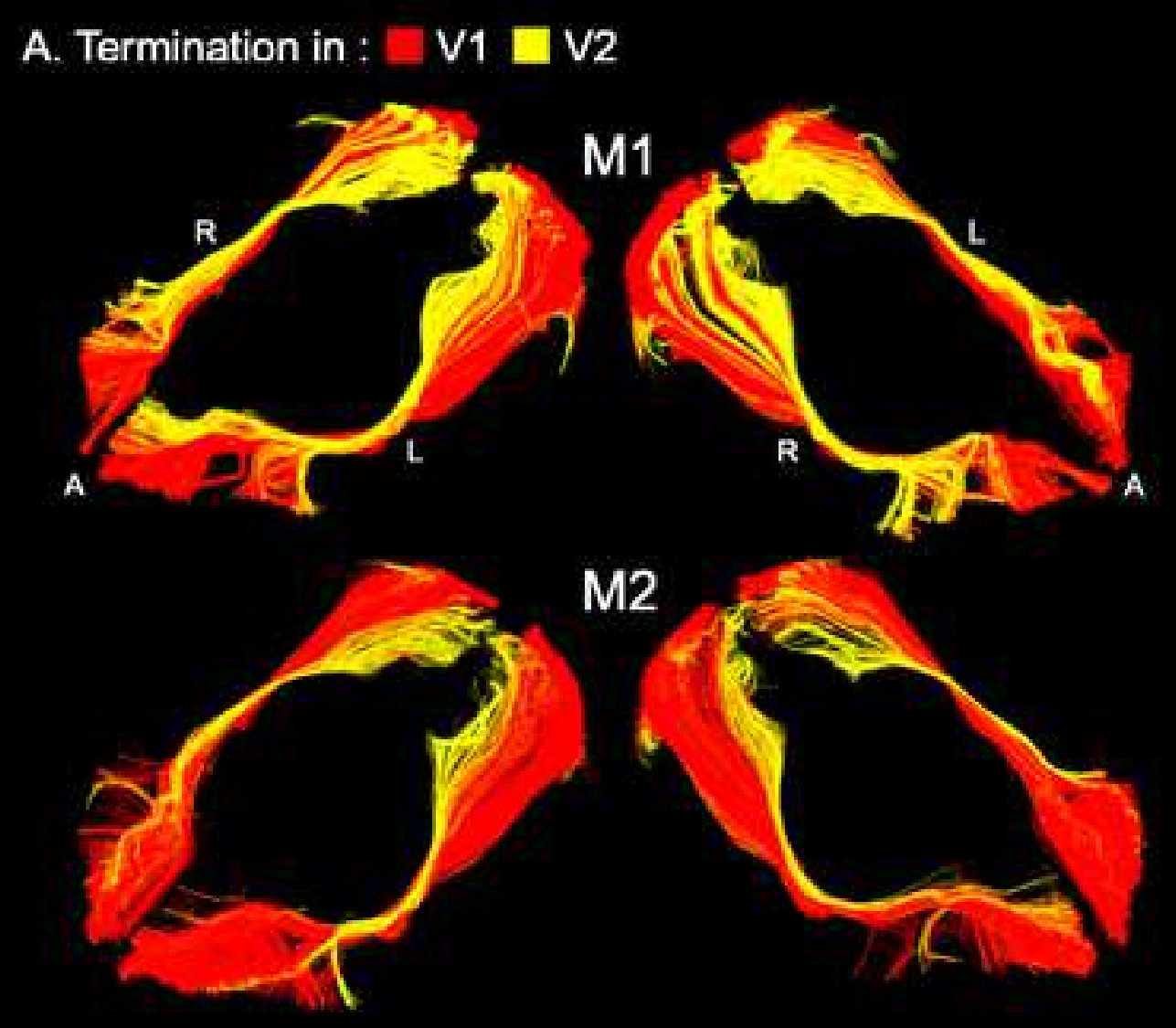

B. Termination in : $\begin{aligned} & \text { Area } 10 \square \text { Area } 11 \\ & \text { Area } 46 \text { Area } 47\end{aligned}$
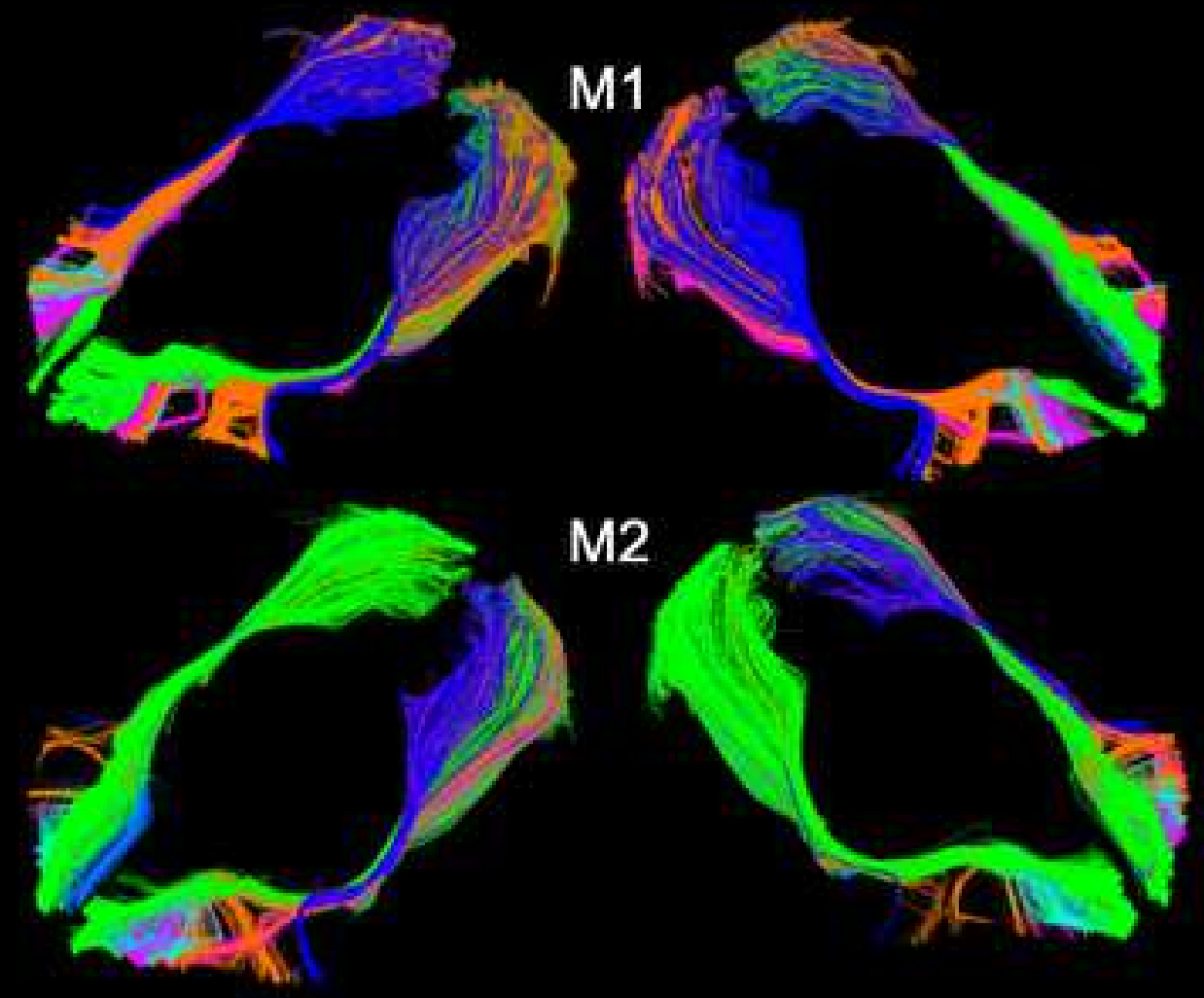


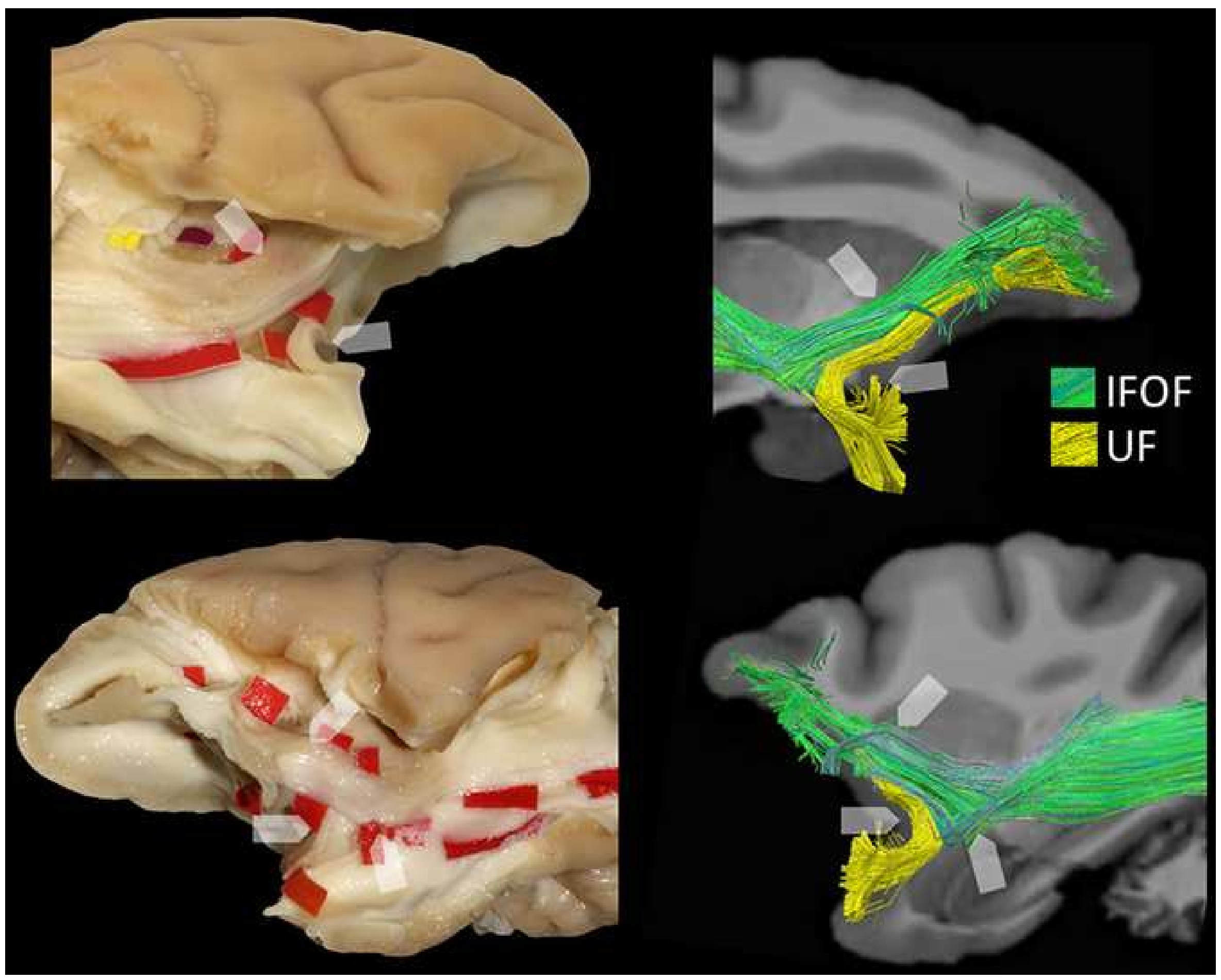




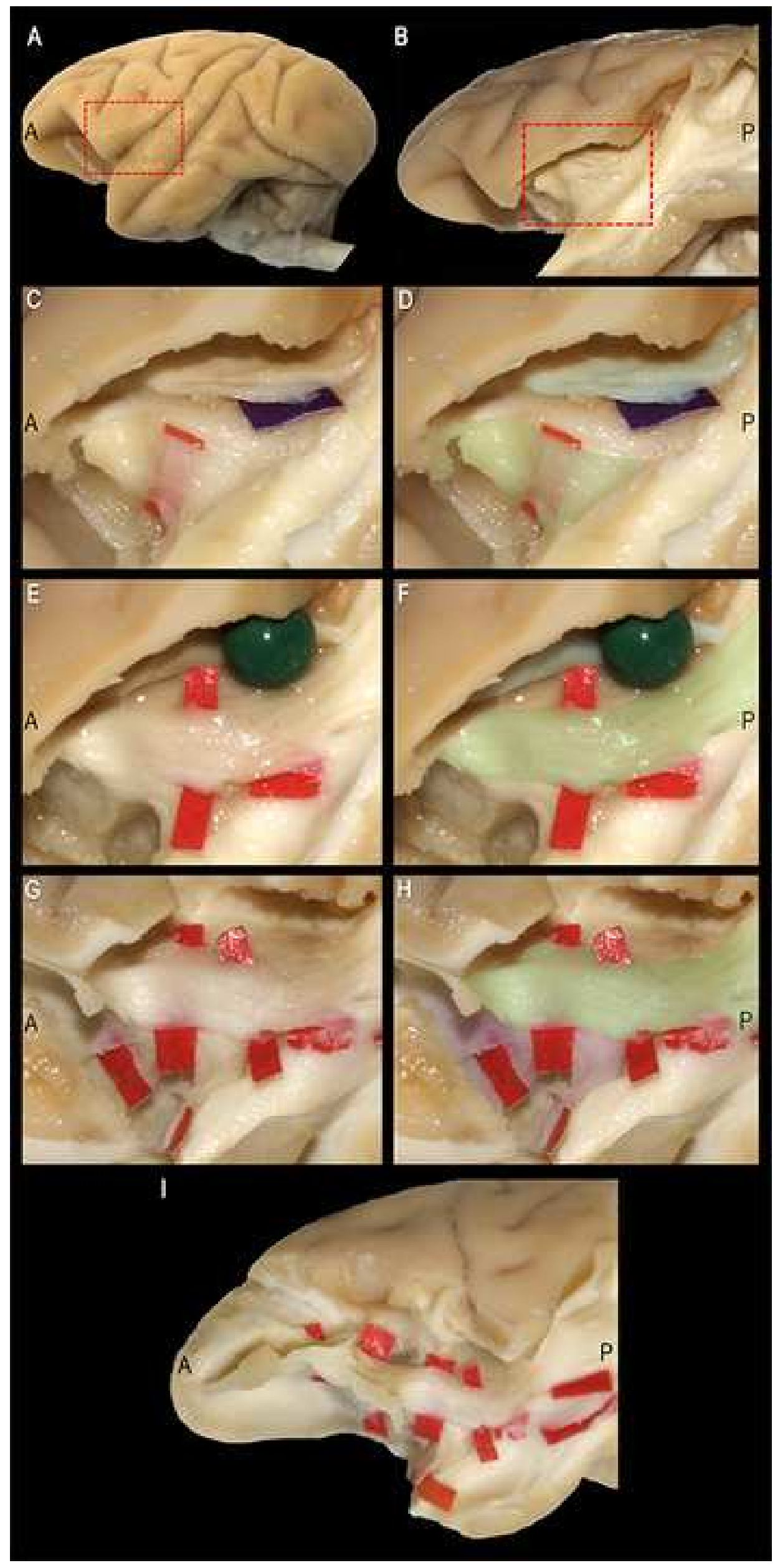




\section{Table 1}

Volumes of the IFOF, ILF, UF and EmC (in $\mathrm{mm}^{3}$ ) as emerged from the tracking of the two hemispheres (left and right) of each scanned brain (M1 and M2). IFOF: fronto-occipital fascicle; ILF: inferior longitudinal fascicle; UF: uncinate fascicle; EmC: extreme capsule.

\begin{tabular}{lllll}
\hline & \multicolumn{2}{c}{ M1 } & \multicolumn{2}{c}{ M2 } \\
& Left & Right & Left & Right \\
\hline IFOF & 1635.2 & 1551.1 & 1339.5 & 1345.6 \\
ILF & 1916.1 & 1926.7 & 1988.2 & 2053.0 \\
UF & 424.0 & 452.4 & 285.6 & 338.9 \\
EmC & 813.5 & 857.7 & 846.1 & 855.6 \\
\hline
\end{tabular}


Table 2. Distribution of the IFOF terminations within the occipital and frontal cortices of the vervet monkey. Area labelling corresponds to the publicly available post-mortem rhesus macaque brain atlas (CIVM) (see Supplementary Table 2 in Calabrese et al. 2015).

\begin{tabular}{|c|c|c|c|c|}
\hline & \multicolumn{2}{|c|}{ M1 } & \multicolumn{2}{|c|}{ M2 } \\
\hline & Left & Right & Left & Right \\
\hline \multicolumn{5}{|c|}{ Occipital IFOF terminations (in \%) } \\
\hline V1 & $\mathbf{7 7 . 8}$ & 61.7 & 91.2 & 90.4 \\
\hline $\mathbf{V} 2$ & 21.5 & 38.0 & 8.6 & 9.5 \\
\hline V3d & 0.7 & 0.3 & 0.2 & 0.1 \\
\hline $\mathrm{V} 3 \mathrm{v}$ & 0.0 & 0.0 & 0.0 & 0.0 \\
\hline V3A & 0.0 & 0.0 & 0.0 & 0.0 \\
\hline \multicolumn{5}{|c|}{ Frontal IFOF termination (in \%) } \\
\hline Area 4 & 0.0 & 0.0 & 0.0 & 0.0 \\
\hline Area 6 & 0.0 & 0.0 & 0.0 & 0.0 \\
\hline Area 8 & 0.0 & 0.0 & 0.2 & 0.0 \\
\hline Area 9 & 0.2 & 0.0 & 0.5 & 0.2 \\
\hline Area 9-46 & 0.0 & 0.0 & 0.5 & 0.1 \\
\hline Area 10 & 36.9 & 2.6 & 50.6 & 85.9 \\
\hline Area 44 & 0.0 & 0.0 & 0.0 & 0.0 \\
\hline Area 45 & 0.0 & 0.6 & 3.8 & 0.2 \\
\hline Area 46 & 1.3 & 0.8 & 4.9 & 1.9 \\
\hline Area 47 & 33.5 & 36.8 & 11.8 & 1.6 \\
\hline Area ProMotor & 17.3 & 38.5 & 9.8 & 0.2 \\
\hline Area 11 & 8.7 & 19.4 & 14.8 & 4.4 \\
\hline Area 13 & 0.2 & 1.2 & 0.0 & 0.0 \\
\hline Area 14 & 1.9 & 0.0 & 2.0 & 5.5 \\
\hline Area 25 & 0.0 & 0.0 & 0.0 & 0.0 \\
\hline Orbital cortex & 0.0 & 0.0 & 1.1 & 0.0 \\
\hline Gustatory Cortex & 0.0 & 0.1 & 0.0 & 0.0 \\
\hline
\end{tabular}




\section{SUPPLEMENTARY MATERIAL}

\section{Uncovering the inferior fronto-occipital fascicle and its topological organization in non-}

\section{human primates: The missing connection for language evolution.}

Silvio Sarubbo ${ }^{{ }^{* \wedge}}$, Laurent Petit $^{2^{\wedge}}$, Alessandro De Benedictis ${ }^{3}$, Franco Chioffi $^{1}$, Maurice Ptito ${ }^{4}$, Tim B. Dyrby ${ }^{5,6}$.

${ }^{1}$ Division of Neurosurgery, Department of Emergency, Azienda Provinciale per i Servizi Sanitari (APSS)

- 38122 Trento, Italy

${ }^{2}$ Groupe d'Imagerie Neurofonctionnelle, Institut des Maladies Neurodégénératives - UMR 5293, CNRS, CEA University of Bordeaux, Bordeaux, France

${ }^{3}$ Neurosurgery Unit, Department of Neuroscience and Neurorehabilitation, Bambino Gesù Children Hospital, IRCCS - 00165 Rome, Italy

${ }^{4}$ École d'optométrie, Université de Montréal, Montreal, QC, Canada

${ }^{5}$ Danish Research Centre for Magnetic Resonance, Center for Functional and Diagnostic Imaging and Research, Copenhagen University Hospital Hvidovre, Hvidovre, Denmark;

${ }^{6}$ Department of Applied Mathematics and Computer Science, Technical University of Denmark, Kongens Lyngby, Denmark

\section{* Corresponding Author}

Silvio Sarubbo, MD PhD

Division of Neurosurgery - Department of Emergency

Structural and Functional Connectivity Lab Project

“S. Chiara" Hospital, APSS Trento

9, Largo Medaglie d'Oro

38122 Trento (Italy)

e-mail: silviosarubbo@gmail.com

${ }^{\wedge}$ These Authors contributed equally 
Fig. 1: Summary of the different steps of post-processing for the extraction of the whole-brain tractogram.
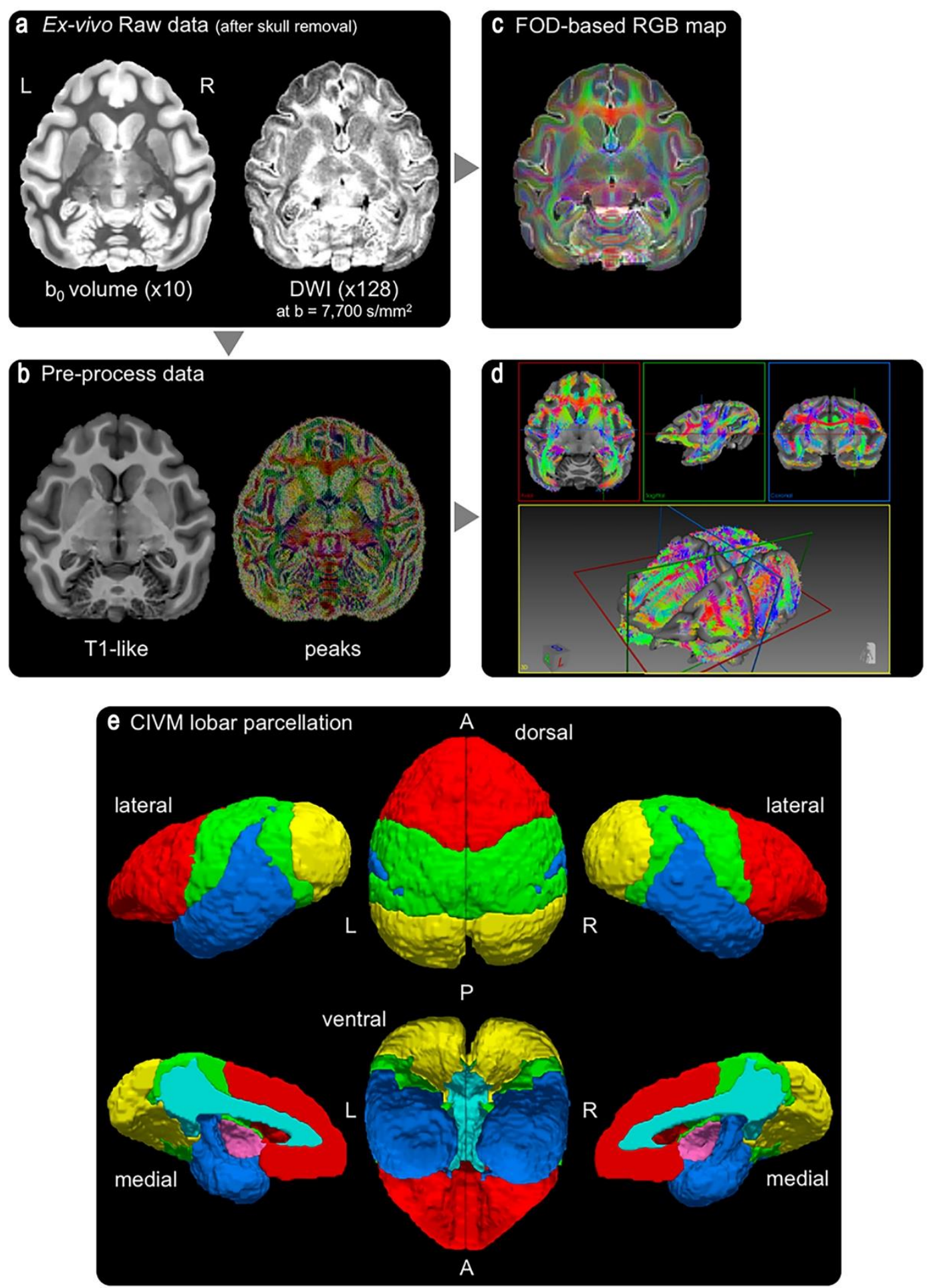
Fig. 2: Summary of the multiple steps for cleaning the whole-brain tractogram.

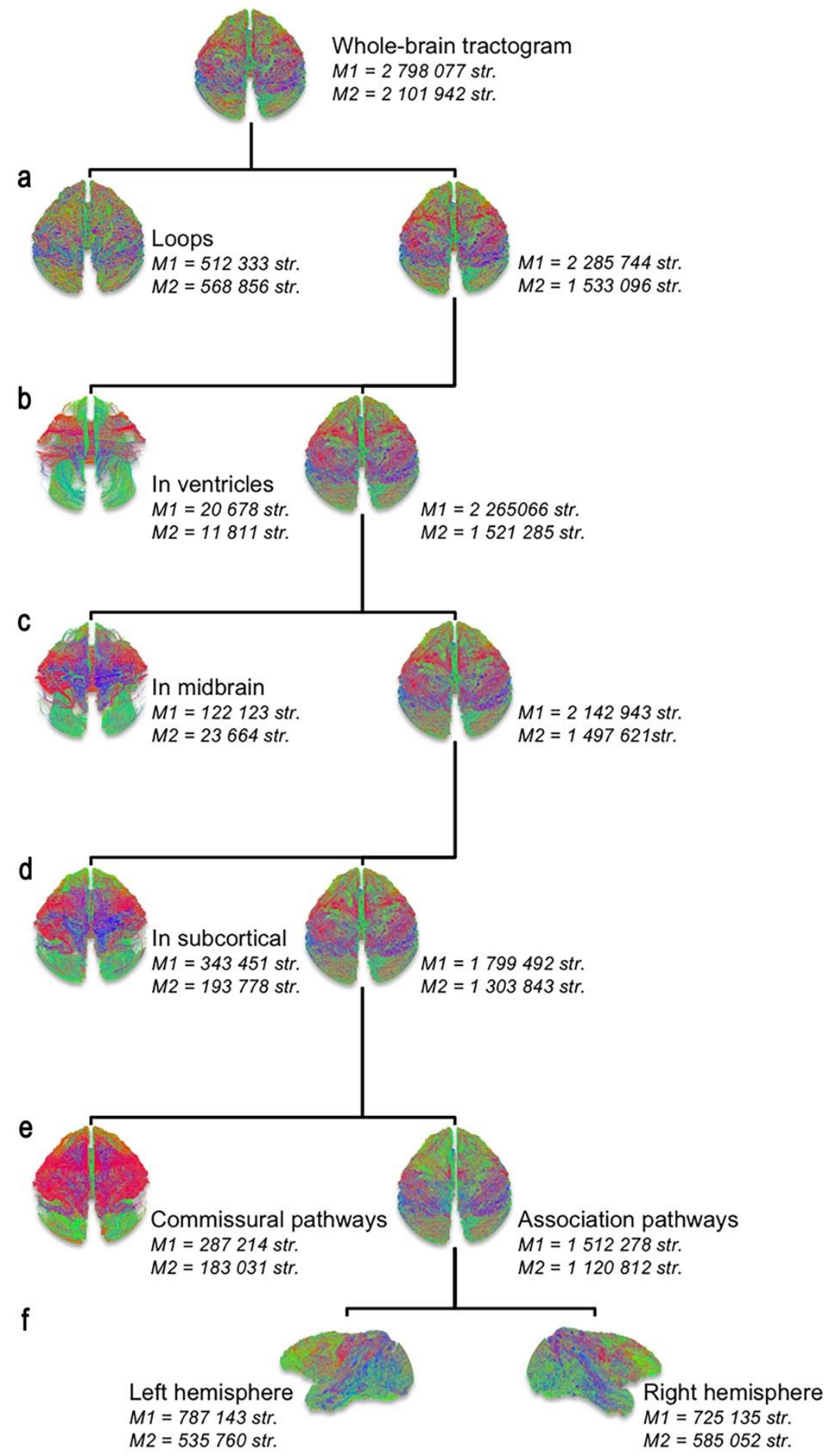

